Time Management Styles and Food Consumption: A Pilot Study of American and Chinese College Students

Tal Gordon and Napatsorn Jiraporn

BRC Journal of Advances in Business 3, no. 1 (2018): 35-49.

http://dx.doi.org/10.15239/j.brcadvb.2018.03.01.ja03

\title{
WEB APPENDIX
}

http://dx.doi.org/10.15239/j.brcadvjb.2018.03.01.wa03 
Figure 1: The Interaction Effect of Culture and Time Organization on Ratio of Healthy Food Choices Selected by Participants

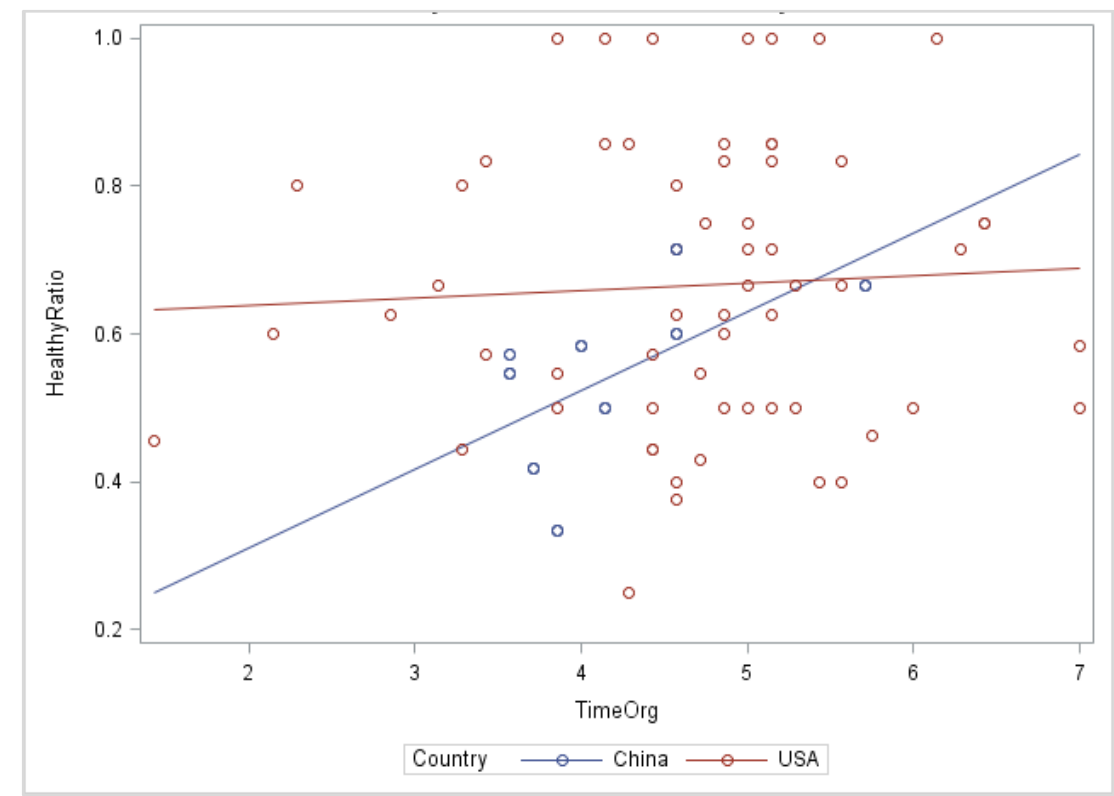

Figure 2: The Main Effect of Preference for Organized Time on Motivation to Select Food based on Health Motives

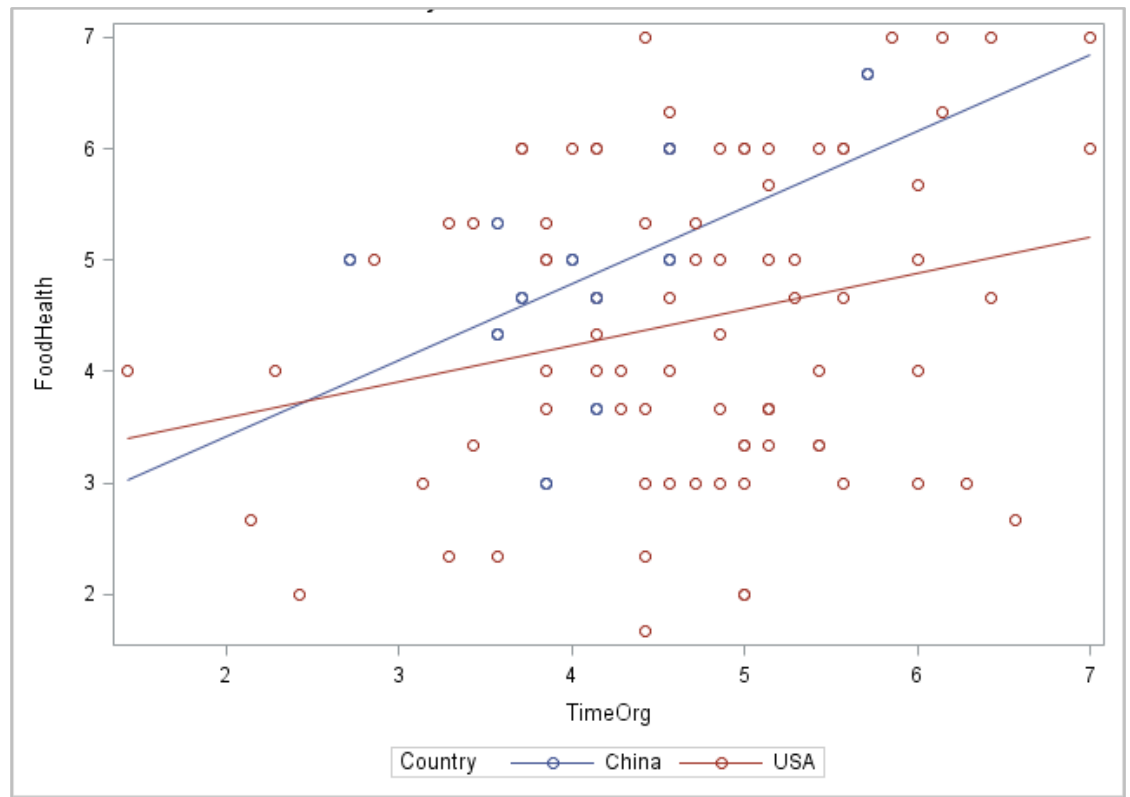


Figure 3: The Interaction Effect of Country and Preference for Organized Time on Motivation to Select Food based on Habits

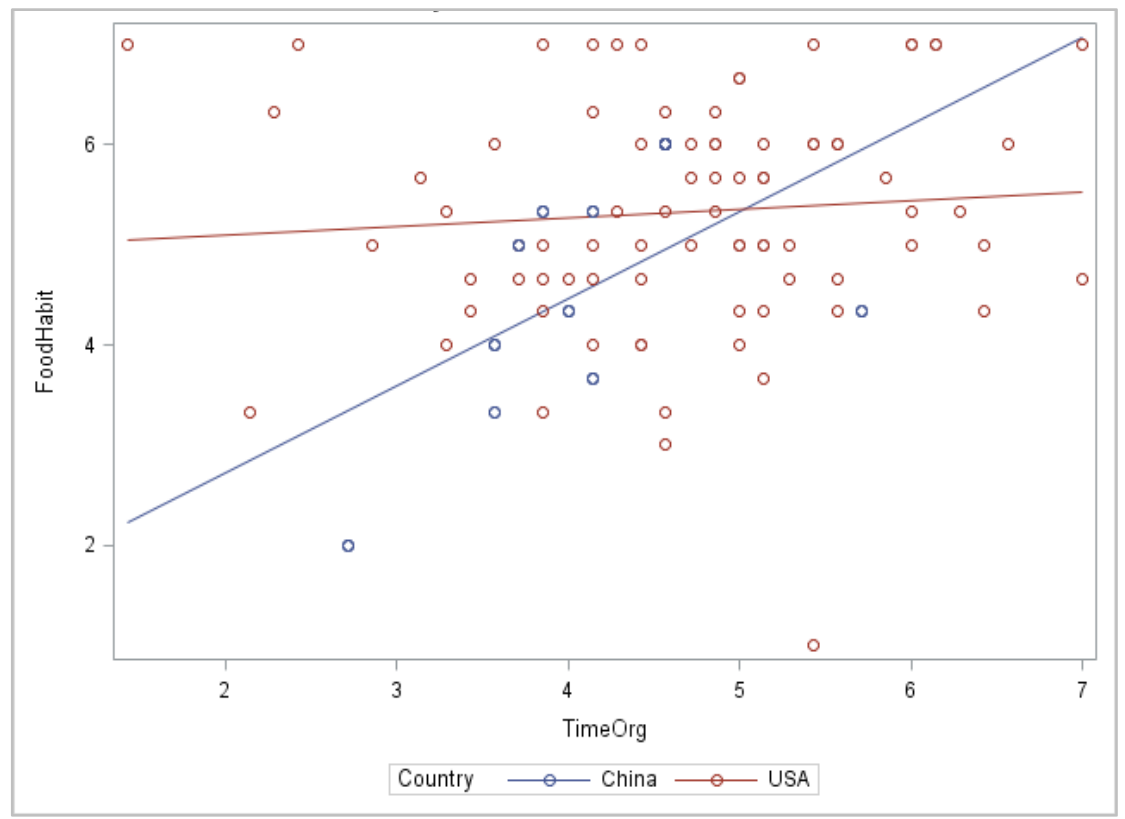


Figure 4: Comparison of Eating Motives between American vs. Chinese College Students (Continued)

I eat what I eat because......

Social Image*

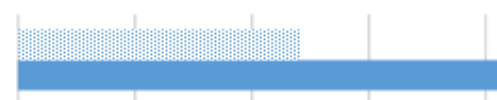

Social Norm*

Weight Control

Visual Appeal

Price

Sociability*

Natural Concerns

Traditional Eating

Pleasure

Convenience*

Health

Hunger

Habits*

Liking*

* p value

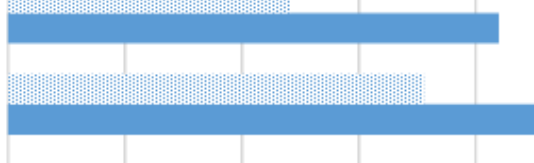

|| || || || || || || || || || || || || || || || || || || || || || ||| ||
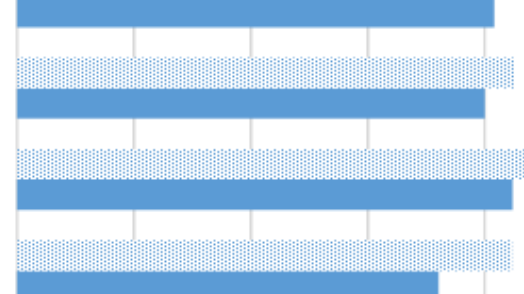

III
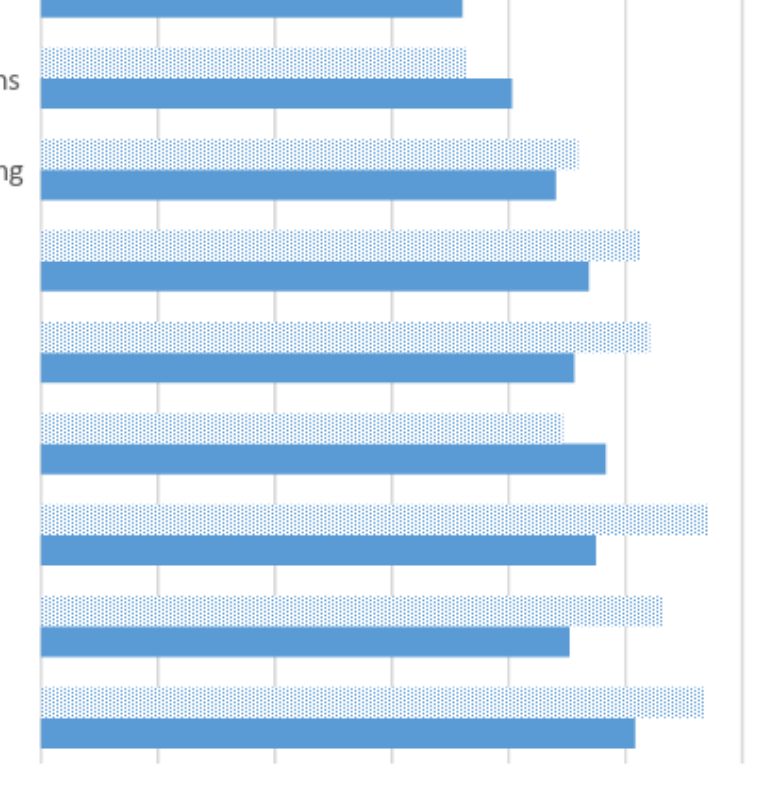

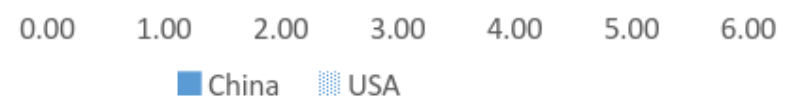

Figure 4: Comparison of Eating Motives between American vs. Chinese College Students

\begin{tabular}{|c|c|c|c|}
\hline $\begin{array}{c}\text { Eating Motives } \\
\text { I eat what I eat because.... }\end{array}$ & China & USA & t value \\
\hline
\end{tabular}




\begin{tabular}{|c|c|c|c|c|}
\hline $\begin{array}{c}\text { Liking: } \\
\text { Because I have an appetite for it } \\
\text { Because it tastes good } \\
\text { Because I like it }\end{array}$ & 5.08 & 5.68 & -3.06 & 0.00 \\
\hline $\begin{array}{c}\text { Habits: } \\
\text { Because I am accustomed to eating it } \\
\text { Because I usually eat it } \\
\text { Because I am familiar with it }\end{array}$ & 4.52 & 5.33 & -3.36 & 0.00 \\
\hline $\begin{array}{c}\text { Hunger: } \\
\text { Because I need energy } \\
\text { Because it is pleasantly filling } \\
\text { Because I'm hungry } \\
\end{array}$ & 4.75 & 5.71 & -5.27 & 0.00 \\
\hline $\begin{array}{c}\text { Health: } \\
\text { I want to maintain a balanced diet } \\
\text { Because it is healthy } \\
\text { Because it keeps me in shape }\end{array}$ & 4.83 & 4.47 & 1.35 & 0.18 \\
\hline $\begin{array}{c}\text { Convenience: } \\
\text { Because it is quick to prepare } \\
\text { Because it is the most convenient } \\
\text { Because it is easy to prepare }\end{array}$ & 4.56 & 5.21 & -2.71 & 0.01 \\
\hline $\begin{array}{c}\text { Pleasure: } \\
\text { Because I enjoy it } \\
\text { In order to indulge myself } \\
\text { In order to reward myself }\end{array}$ & 4.69 & 5.13 & -1.92 & 0.06 \\
\hline $\begin{array}{c}\text { Traditional Eating: } \\
\text { Because it belongs to certain situations } \\
\text { Out of traditions } \\
\text { Because I grew up with it }\end{array}$ & 4.41 & 4.59 & -0.76 & 0.45 \\
\hline $\begin{array}{c}\text { Natural Concerns: } \\
\text { Because it is natural } \\
\text { Because it contains no harmful substances } \\
\text { Because it is organic }\end{array}$ & 4.03 & 3.64 & 1.23 & 0.22 \\
\hline $\begin{array}{c}\text { Sociability: } \\
\text { Because it is social } \\
\text { So that I can spend time with other people } \\
\text { Because it makes social } \\
\text { It makes social gatherings more comfortable }\end{array}$ & 3.60 & 4.24 & -2.35 & 0.02 \\
\hline $\begin{array}{c}\text { Price: } \\
\text { Because it is inexpensive } \\
\text { Because I don't want to spend any more money } \\
\text { Because it is on sale }\end{array}$ & 4.24 & 4.54 & -1.05 & 0.29 \\
\hline $\begin{array}{c}\text { Visual Appeal: } \\
\text { Because the presentation is appealing } \\
\text { Because it spontaneously appeals to me } \\
\text { Because I recognize it from advertisements or have seen it on TV }\end{array}$ & 4.00 & 4.26 & -0.96 & 0.34 \\
\hline $\begin{array}{c}\text { Weight Control: } \\
\text { Because it is low in calories } \\
\text { Because I watch my weight } \\
\text { Because it is low in fat }\end{array}$ & 4.08 & 3.93 & 0.49 & 0.63 \\
\hline $\begin{array}{c}\text { Social Norm: } \\
\text { Because it would be impolite not to eat it } \\
\text { To avoid disappointing someone who is trying to make me happy } \\
\text { Because I am supposed to eat it }\end{array}$ & 4.54 & 3.56 & 3.23 & 0.00 \\
\hline $\begin{array}{c}\text { Social Image: Because it is trendy } \\
\text { Because it makes me look good in front of others } \\
\text { Because others like it }\end{array}$ & 4.20 & 2.41 & 6.70 & 0.00 \\
\hline
\end{tabular}

